\title{
A Battery Certification Testbed for Small Satellite Missions
}

\author{
Zachary Cameron*, Chetan S. Kulkarni ${ }^{\dagger}$, Ali Guarneros Luna ${ }^{\ddagger}$, Kai Goebel ${ }^{\ddagger}$, and Scott Poll ${ }^{\ddagger}$ \\ *Dept. of Aerospace, Pennsylvania State University, PA 16801 USA \\ zac5060@psu.edu \\ †SGT Inc., NASA Ames Research Center, Moffett Field, CA 94035, USA \\ chetan.s.kulkarni@nasa.gov \\ ${ }^{\ddagger}$ NASA Ames Research Center, Moffett Field, CA 94035, USA \\ ali.guarnerosluna@nasa.gov, kai.goebel@nasa.gov, scott.poll@nasa.gov
}

\begin{abstract}
A battery pack consisting of standard cylindrical 18650 lithium-ion cells has been chosen for small satellite missions based on previous flight heritage and compliance with NASA battery safety requirements. However, for batteries that transit through the International Space Station (ISS), additional certification tests are required for individual cells as well as the battery packs. In this manuscript, we discuss the development of generalized testbeds for testing and certifying different types of batteries critical to small satellite missions. Test procedures developed and executed for this certification effort include: a detailed physical inspection before and after experiments; electrical cycling characterization at the cell and pack levels; battery-pack overcharge, over-discharge, external short testing; battery-pack vacuum leak and vibration testing. The overall goals of these certification procedures are to conform to requirements set forth by the agency and identify unique safety hazards. The testbeds, procedures, and experimental results are discussed for batteries chosen for small satellite missions to be launched from the ISS.
\end{abstract}

\section{INTRODUCTION}

The International Space Station (ISS) plans to launch $100+$ small-sat missions for different science experiments in the next coming years [1]. Many of these missions will be powered by standard off-the-shelf cylindrical 18650 lithium-ion cells in their current and updated forms as technology improves. A battery pack consisting of lithium-ion cells has been chosen to fly based on previous flight heritage and compliance with NASA battery safety requirements. Before batteries can be used for small satellite missions on ISS, both the individual cells as well as packs of multiple cells must be certified for safe operations. Certification tests for the cells and packs include electrical cycling characterization, over charging/discharging, external shorting, vibrational excitation, and exposure to vacuum. Only after each cell and pack has passed these certification tests can the batteries be installed in cube sats and delivered to the ISS.

Dependable, energy dense, and durable power sources are critical components for small satellite missions. They are subjected to the same strenuous operating conditions that the satellite is subjected to during transit to the ISS, deployment into space, and for the duration of the mission after launch from the ISS. Since the batteries come into close proximity to the astronauts on the ISS, it is critical to establish rigorous testing procedures to certify their safety.

Normal operation requires repeated charging and discharging of the batteries that age the packs and can lead to activation of internal fault protection systems. These critical internal fault protection systems prevent the batteries from destructively failing in adverse scenarios, and protect electronic equipment from becoming damaged.

Through regular cycling of lithium-ion batteries from full charge to low charge, the battery ages, losing the ability to hold full charge and to recharge the same amount. Through proper use and high quality construction, lithium-ion batteries can survive hundreds to thousands of these cycles depending on their operating conditions. In order to properly charge and discharge fully and efficiently, it is important to understand the batteries' charging characteristics. Cycling the batteries from maximum charge to minimum charge provides valuable information on the batteries, health and ability to recharge. This cycling also ensures that the batteries perform as specified and can be expected to perform appropriately when in operation.

One of several adverse scenarios batteries may experience is unintentional battery shorting. Lithium-ion batteries pose a risk of sharp temperature increases when shorted, to the point of chemical and electrical failure. This risks melting the battery and surrounding components and expelling hazardous and corrosive gasses. Internal short prevention in lithium-ion batteries effectively opens the battery circuit, shutting off current flow through the battery. This prevents damage to the battery 
and allows for possible future battery usage if the short can be corrected or if the cell removed from operation. In the 18650 lithium-ion cells, a PTC (Positive Temperature Coefficient) switch is used to prevent short-circuiting by inhibiting high current surges. This occurs via a large increase in material resistance when the temperature exceeds a threshold limit. The resistance can increase to hundreds of thousands of ohms, which inhibits the current from flowing from the battery. PTC switches are reversible, and will cycle back to a conductive state when the short circuit is removed, but they can suffer in reliability when material standards are not upheld [2], [3]. Despite having PTC switches installed, some 18650 lithium-ion batteries still fail due to high currents and overheating, giving purpose to certification standards.

Another unfavorable condition leading to faults in the cells is over charging/discharging. The ability to prevent such damaging cycling protects sensitive electronic systems powered by the batteries from being exposed to high or low voltages that could terminate operation. It also prevents over pressurization of the battery cells while overcharging, and chemical aging resulting from over discharging. CIDs (Current Interrupter Devices) are capable of reversibly opening the positive battery terminals circuit and preventing further charging of a battery until internal pressure is alleviated [3]. These devices prevent venting of hazardous electrolytes and bursting due to buildup of high pressures.

Payloads aboard rockets are subjected to vibratory loads over a large bandwidth of frequencies. These vibrations pose a threat to internal and external structural stability, can cause exterior leaks, internal shorting of the batteries, and damage to protection circuits. Small-sat missions are required to withstand prolonged exposure to the vacuum of space. Electrolytic material is contained in the battery using a simple crimp in the outer metal shell of the batteries. Low quality crimp seals can allow electrolytic material to leak in low-pressure environments, diminishing battery charge and life. Certification of the crimp is critical for ensuring that individual cells will not leak after launching from the ISS.

Vacuum leak tests are conducted to observe any leaks from the cell under very low-pressure conditions to detect any manufacturing faults that may lead to operational failures of the batteries. A vibration testbed is developed to conduct certification for physical connectivity in the pack circuits and well as full charge battery operation under launch and deployment environment during which the batteries undergo maximum vibration loads. A prolonged electrical short test is conducted to observe the activation of safety circuit in the battery packs to avoid any fire hazard. We will discuss each of the developed testbeds in later sections of the paper and also discuss the test results observed.

The structure of the paper is as follows. Section II discusses the safety related requirements for space flight batteries. Section III describes different fault modes that may lead to accidents depending upon usage and abusive operating conditions. Section IV discuss the certification testbed setup, tests conducted and some results. The paper ends with conclusions and future work in Section V.

\section{SAFETY}

The main abuse conditions that cause hazards in lithium-ion cells are overcharge, external and internal short circuits, over-discharge, high temperatures, and structural issues.

Safety is the key aspect of any energy storage device, including batteries. It is important to understand that batteries contain both oxidizer (anode) and fuel (cathode) in a sealed container. Under normal operations, the fuel and oxidizer convert the chemical energy to electrical energy with minimal heat and negligible gas [4]. If allowed to react chemically in an electrochemical cell, the fuel and oxidizer convert the chemical energy directly into heat and gas. Once started, chemical reaction will likely proceed to completion [5].

The battery safety approval process for all payloads is conducted by a duly designated Payload Safety Review Panel (PSRP). This process should be considered in the early stages of the payload design process, so that the PSRP and other teams can agree upon a process specific to the anticipated battery chemistry and payload power requirements. One of the key requirements for an acceptable battery design is to include controls for any potential battery hazards [6]. An acceptable battery design should consider structural integrity of the cell and battery housings, the likelihood of gas generation, pressure or electrolyte leakage, the prevention of short circuits, probability for high battery temperatures, overdischarging, and ability of efficient charging methods. The battery evaluation will assess the battery hazard controls which are dependent on parameters such as battery chemistry, capacity, applications, etc.

Safety needs have to be addressed at the cell, pack, device and ultimately at the mission level. Failure at low levels can have a cascading effect at higher levels which directly affects overall mission safety. It is difficult to define safety by a single parameter. A set of approaches is combined together to enhance safety by a) reducing the probability of an unsafe event, b) reducing potentil of harm to personnel, c) minimizing the effect of an unsafe event on mission completion and safety [6]. 


\section{Typical Failure Modes}

Although there are some common failure modes despite different chemistries, some of the failure modes are unique to individual chemistries [5]. When batteries are operated beyond their nominal operating conditions, generation of excessive heat can lead to faults. It is very important to understand the limitations of the battery chemistry and probable operating conditions of the mission. Some of the failures observed in lithium-ion batteries are discussed below.

\section{A. Charge Discharge Failures}

Failures due to overcharge and over-discharge can occur if either the internal electronics on the battery or control electronics on the battery pack malfunction. The capability of a battery cell or pack to resist overcharge depends on the charging current level as well as specific chemistry of the battery. The reaction to any overcharge condition depends on factors such as control electronics, current, maximum voltage, thermal environment and chemistry. [7]. During a charge cycle, lithium is removed from the cathode oxide material. If the battery cell is charged beyond the limit threshold lithium continues to be removed from the cathode structure, leading to permanent crystallographic changes and increased oxidation potentials [5]. It has been observed that there is an instantaneous increase in heat generation when all the lithium ions are removed from the cathode structure.

\section{B. External Short}

External short is one of the most frequent abusive conditions a cell may undergo during operation. Large cells that can carry higher short circuit currents may cause violent cell venting and thermal runaway condition leading to accidents. An external short under excessive thermal conditions could lead to thermal runaway condition in the cells [8].

\section{Internal Short}

Internal short faults have been studied due to accidents reported during transportation [3], [9]. These accidents could be caused due to any of the following conditions:

- If the manufacturing quality control is not stringent there is a relatively high probability of accidental internal cell shorts due to the presence of impurities such as metal particles, burrs, etc.

- During transportation any foreign object debris or native contaminants during manufacturing could manifest themselves, leading to a unwanted mishap.

- Dendrites are formed inside the cells when they are subjected to voltages, currents and temperatures when operated beyond the manufacturers recommendation.

- Dendrites are also formed due to localized heating or large thermal gradients within a battery module.

- Incorrect cell design may lead to dry out of the electrode in certain areas of the cell, which may lead to internal shorting.

\section{Certification Testbeds and Results}

Experiments used off-the-shelf 18650 cells rated at $2100 \mathrm{~mA}$. A battery pack consisted of 2 cells in series, which were in parallel with another set of 2 cells in series. A LITH-X ${ }^{T M}$ fire extinguisher powder is kept close to the testbeds in case any emergency situation arises during the electrochemical and external short procedures. Table I notes the different roles and responsibilities of each of the personnel associated with the certification process. The processes that have to be followed during each of the certification tests are discussed later in the section.

\section{A. Electrical Cycling Characteristics}

In this section we discuss the setup for the electrochemical characterization of the batteries. In the electrical cycling characterization procedure, cells and packs are subjected to flight charge/discharge profiles. The battery packs are subjected to different operating conditions to test the safety mechanisms under adverse conditions. The tests are conducted using a MACCOR 4000 shown in Fig. 1, which is a battery tester that allows for a range of fully automated, computerized tests. The steps for preparing the battery cells for the experiment are as follows:

- Record the $\mathrm{S} / \mathrm{N}$ for each battery cell on the physical and electrochemical characteristics log.

- Record dimensions of all cells using a calibrated caliper.

- Record weight of all cells.

- If the cell voltage is below $4.0 \mathrm{~V}$, then recharge the cells before performing the closed circuit voltage (CCV) test.

$\mathrm{CCV}$ tests are performed on all cells using a $1.0 \mathrm{C}$ current for 30 seconds. Current, voltage, and temperature measurements are recorded for all tests. The cells are charged using the Constant Current/Constant Voltage $(\mathrm{CC} / \mathrm{CV})$ protocol and discharged as follows:

- Measure and record Open Circuit Voltage (OCV) of battery packs.

- Charge the cells using a C/2 current to $4.2 \mathrm{~V}$ and then hold at a constant voltage of $4.2 \mathrm{~V}$ until the current drops to about $50 \mathrm{~mA}$. 
TABLE I

ROLES AND RESPONSIBILITY TABLE OF NODES BATTERIES BUILD PROCEDURES

\begin{tabular}{|c|c|c|}
\hline Position & Role & Responsibilities \\
\hline Engineer/Technician & Operator & The Engineer/Technician performs the procedures. \\
\hline Operations Engineer & Inspector & The Operations Engineer inspects the final product of the Engineer/Technician. \\
\hline Quality Assurance Representative & Observer & $\begin{array}{l}\text { Representative ensures procedures are followed, safety concerns are mitigated, and } \\
\text { provides confirmation stamps at required points. }\end{array}$ \\
\hline
\end{tabular}

- Provide a rest period of 10 minutes after charge.

- Discharge the cells using a $\mathrm{C} / 2$ current to a cutoff voltage of 3.0 V. A pulse shall be performed at the midpoint of the discharge with a $3.0 \mathrm{C}$ or $1.5 \mathrm{C}$ current for $100 \mathrm{~ms}$.

- Provide a rest period of 15 minutes after discharge.

- Record current, voltage and temperature during the cycles in the log file.

Similar steps are followed for the electrochemical characteristics of the battery packs. If the battery voltage is below $8.0 \mathrm{~V}$, then recharge the cells and battery packs before performing the $\mathrm{CCV}$ test. If the voltage of the battery pack falls below $6.0 \mathrm{~V}$, then it is recharged and the $\mathrm{OCV}$ is measured and recorded. The CCV test is performed on all battery packs using a $1.0 \mathrm{C}$ current for 30 seconds. All battery packs undergo two charge/discharge cycles before they are subjected to the tests for safety evaluation of battery packs. Current, voltage, and temperature measurements are recorded for all tests. The protocols followed for these battery pack cycles are provided below:

- Charge and discharge the battery pack using the cell protocol in section above (with $\mathrm{C} / 2$ charge and discharge rates).

- Record voltage at which the MOSFET switch activates.

- Record current, voltage and temperature during the cycles are recorded through the MACCOR test unit.

\section{B. Over-charge and Over-discharge}

Battery pack overcharge/overdischarge tests are discussed in this section. These tests are required to characterize and confirm the safety of the lithium-ion cells and battery packs that are to be used in flight satellite battery systems. In this test we measure current and voltage of the battery pack and temperature of the individual cells. Cells and batteries are capable of venting electrolyte leading to thermal runaway conditions and ultimately causing fire, when they operate beyond their normal operating range. Hence all safety tests shall be carried out in an abuse chamber and the necessary precautions taken to prevent injury to personnel and damage to equipment. The over charge test intends to characterize

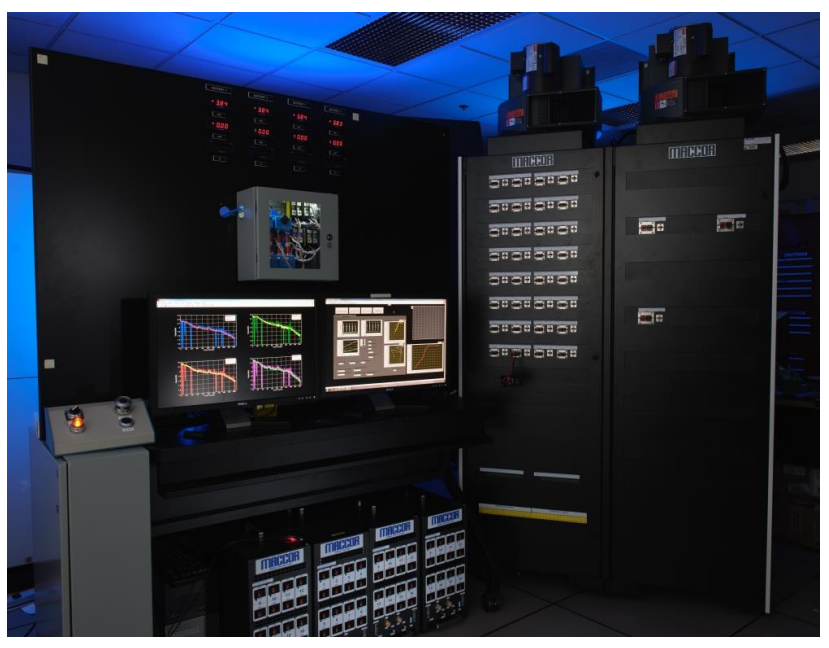

Fig. 1. Electrical Testbed

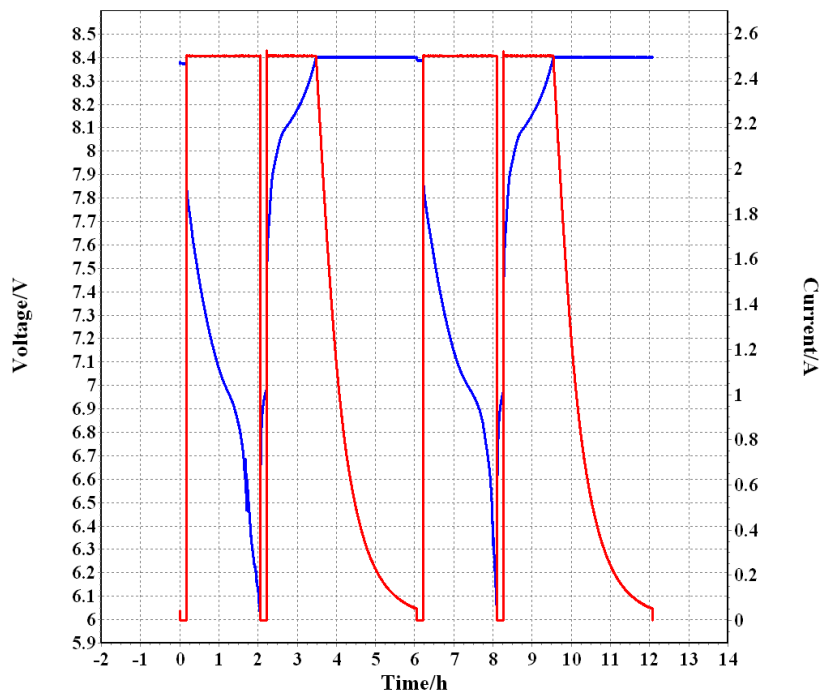

Fig. 2. Sample plot for Electrical Cycling Profile 


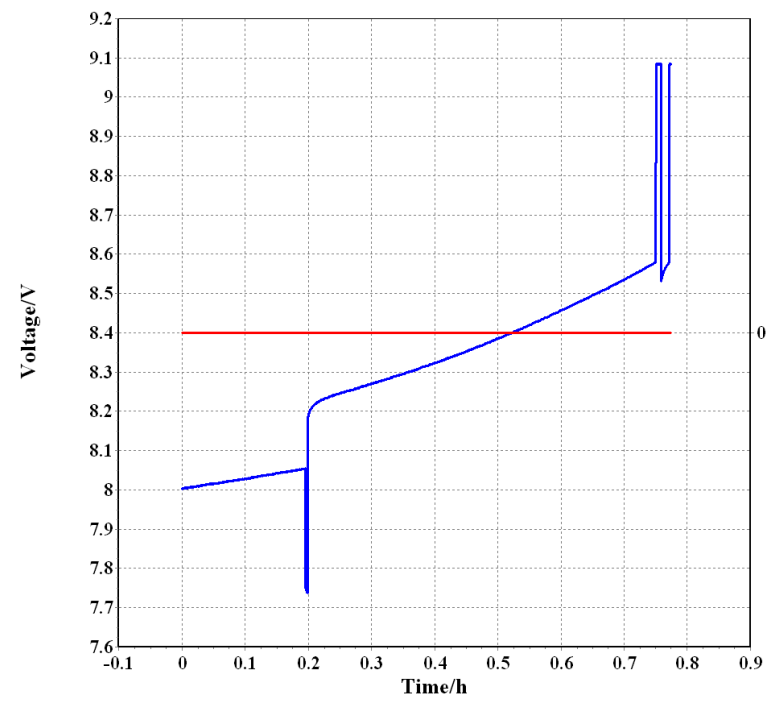

Fig. 3. Sample plot for Over-Charge Test Profile

and confirm the overcharge protection device in the battery. The following steps are followed:

- Record battery S/N and battery cells S/N.

- Overcharge a fully charged battery pack with protective circuit board to a total voltage of $9 \mathrm{~V}$ with a $1.0 \mathrm{C}$ current until the test is stopped due to a high resistance caused by MOSFET activation or a maximum of 6 hours.

- Record the voltage at which the MOSFET switch activates in the log sheet. A sample plot of the test shown in Fig. 3.

- Record current, voltage and temperature for all the tests.

Similarly an over-discharge test is run to characterize and confirm the over-discharge protection device in the battery.

- Record battery S/N and battery cells $\mathrm{S} / \mathrm{N}$.

- Over-discharge a battery pack with protective circuit board with a $3.0 \mathrm{C}$ current to $0 \mathrm{~A}$. The safety MOSFET should have activated by this point.

- Report the voltage at which the safety MOSFET activates in the log.

- Record current, voltage and temperature for all the tests as shown in plot of Fig. 4.

The internal circuit board should prevent the battery pack from going into a reversal condition.

\section{External Short}

This section describes the procedures needed to prepare battery cells and packs for the external battery short procedure. This procedure is conducted as a next

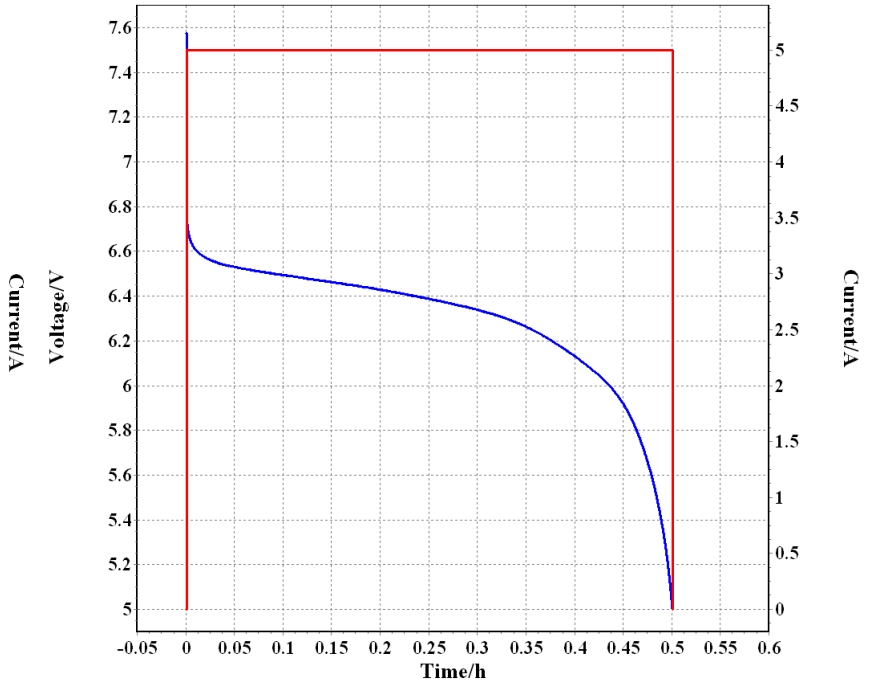

Fig. 4. Sample plot for Over-Discharge Test Profile

step after having passed the physical and electrochemical characteristics tests discussed earlier in the section.

For consistency, a $10-\mathrm{m} \Omega$ circuit is used as a short for testing. It is necessary to ensure that this circuit would be able to withstand high currents and heat that the temporary short, and possibly failed battery extended short would create. The current generated by the short can be calculated using Ohms law:

$$
I_{s}=V_{b} / R_{s}
$$

where $I_{s}$ is current from short, $V_{b}$ is test battery voltage, and $R_{s}$ is the $10 \mathrm{~m} \Omega$ short circuit resistor. The current flow experienced during the short test increases linearly with voltage and requires heavy gage wiring to prevent melting.

The test fixture was encased in a metal enclosure, as shown in Fig.5, to prevent high temperature or possible flame or smoke from escaping a controllable area. Within this enclosure two relays are used for controlling the test, clips to attach the battery to the external short and measurement systems, a thermocouple to monitor the battery and short temperature, and a wire block to act as a stress reliever for wiring. When the short was activated from a control computer using the low resistance relay, the voltage spike and subsequent drop, as well as calculated current spike and drop were examined and recorded. The drop was measured using a picometer connected through $\mathrm{PC}$ and recorded to the microsecond scale as shown in Fig. 6. This allows for a high-resolution image of the activation of the PTC circuit and prevention of extended 


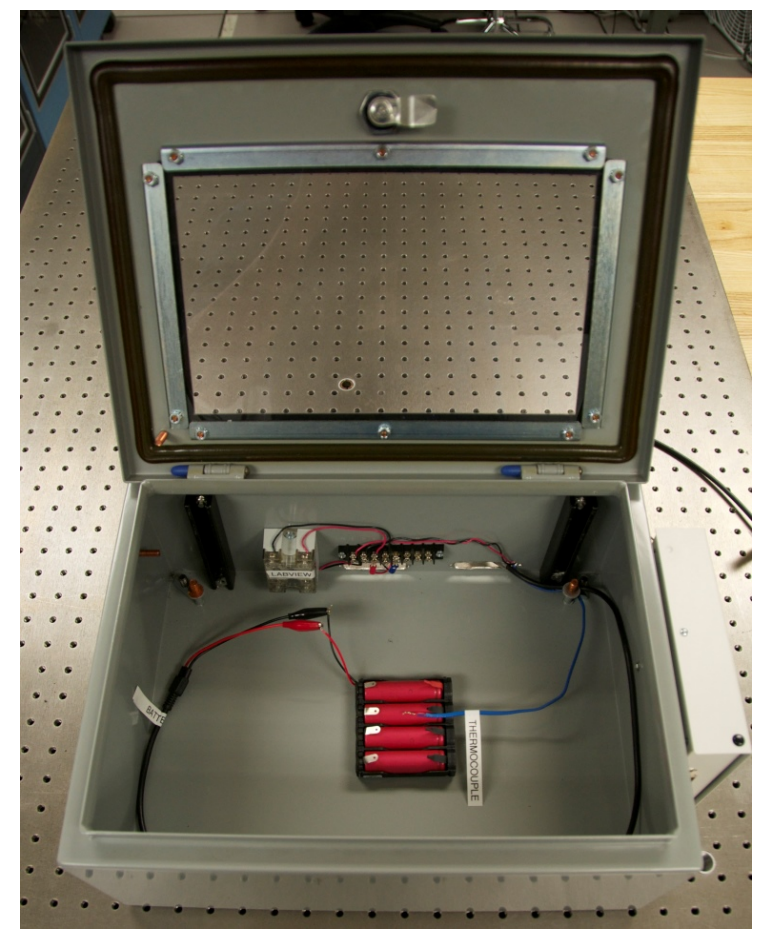

Fig. 5. External Short Testbed

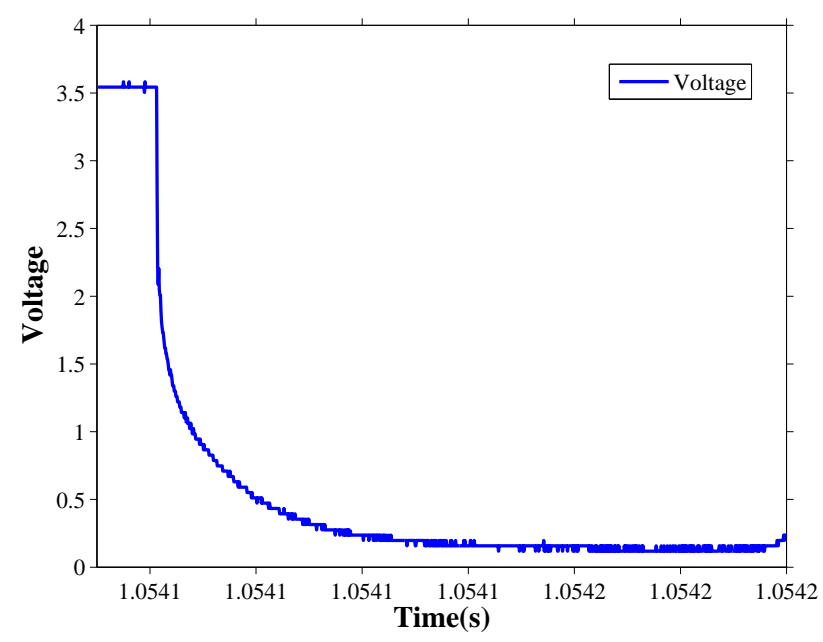

Fig. 6. A sample plot of the initial time frame capture for Short Test Procedure

shorting. For the PTC circuit to be considered fully operational, the short must hold the circuit voltage and current to zero, and maintain those levels for the total test duration of 6 hours. Once this time period was completed and the battery showed no signs of re-initialization of shorting, the test was ended and the battery declared to have passed the test.

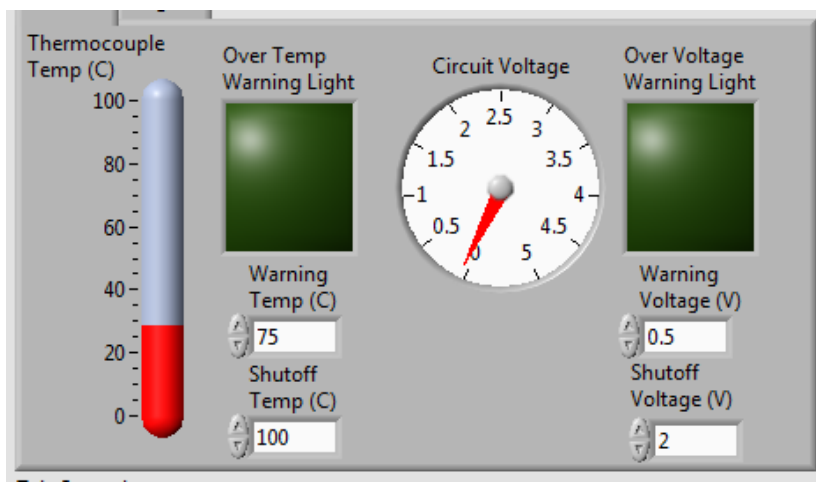

Fig. 7. Developed GUI for Short Test Procedure

In case the MOSFET does not reset itself, a destructive physical analysis of the battery pack is performed to determine cell integrity. The battery packs are opened up and the performance and safety of each cell confirmed by removing them from the pack and subjecting them to charge/discharge cycling. The charge/discharge protocol for cells is as discussed in Section IV-A. We recorded current, voltage and temperature of the battery pack and cells through the data acquisition system as shown in Fig. 7. If the over-current protection in the battery pack does not activate as expected, the battery design shall not be used for flight applications and may require a redesign and retest to confirm that the updated design provides external short protection.

\section{Vacuum Test}

The ISS is the launch platform for small-sats, which orbits at an altitude of $400 \mathrm{~km}$ above Earth. While the boundary between Earth and space is defined as the Karman line at $100 \mathrm{~km}$ above Earth, there are gaseous particles extending to $10,000 \mathrm{~km}$. The ISS still experiences drag due to Earth's atmosphere at its orbital altitude. Air pressure at sea level is approximately 760 Torr, and decreases exponentially with altitude. Using the barometric formula one can provide a reasonable estimate of pressure versus altitude. The pressure estimate is limited to about $100 \mathrm{~km}$ when not accounting for change in temperature with altitude.

$$
\left.P=P_{b} *\left[T_{b} /\left(T_{b}+L_{b} *\left(h-h_{b}\right)\right)\right]^{(}\left(g_{\theta} * M\right) /\left(R^{*} * L_{b}\right)\right)
$$

$\mathrm{P}$ is the calculated pressure at altitude $h$, with $P_{b}$ and $h_{b}$ representing reference pressure and altitude. $L_{b}$ is the standard temperature lapse rate, $g_{\theta}$ is gravitational acceleration, and $\mathrm{M}$ is the molar mass of Earth's air. Using equation 2 the pressure is estimated to be approximately 0.001 Torr, or $0.0001 \%$ of atmospheric pressure. 
Based upon previous tests and the need for a streamlined, reliable and repeatable testing procedure, a pressure of 5 Torr was chosen as the testing threshold pressure in the chamber seen in Fig. 8. While not equivalent to the near complete vacuum present at the altitude of the ISS, 5 Torr $(0.1 \mathrm{psi})$ is roughly $0.65 \%$ of atmospheric pressure at sea level. This pressure is much more manageable to create and maintain in a laboratory setting consistently, and based upon previous results provides adequate near vacuum conditions to replicate conditions subjected to the batteries in deployment. Batteries are held at this pressure to test the crimped seal at the battery cap for electrolytic leaks. After the test chamber reaches 5 Torr, it is held at constant pressure, and a constant temperature of $25^{\circ} \mathrm{C}$ for 6 hours.

The mass of the batteries is measured before and after vacuum exposure to the thousandth of a gram to examine any potential electrolyte leaks through the external sleeve of the cell. If the total change in mass of the batteries exceeds $0.25 \%$ the batteries are considered a failure and unfit for deployment. In addition the operational capability of the pack is validated by running the charge/discharge profile discussed in section IV-A.

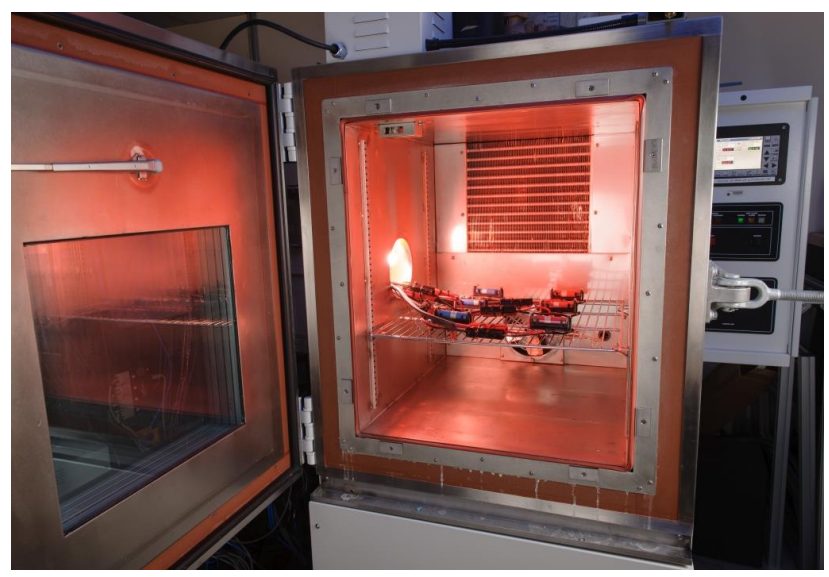

Fig. 8. Vacuum Chamber

\section{E. Vibration Test}

In transit to the ISS the cube-sats will be subject to high vibrational levels created by the various stages of the delivery rocket. The levels of vibrations the payloads will experience are dependent upon various factors such as the number of stages, the rocket size, the type of rocket, and the method with which the payload is attached.

Currently payloads reach the ISS through rocket launches of different providers. While payload vibration exposure is partially dependent upon payload weight, a generalized set of frequencies at certain acceleration levels can be defined to help simulate similar responses through the testbed shown in Fig. 9.

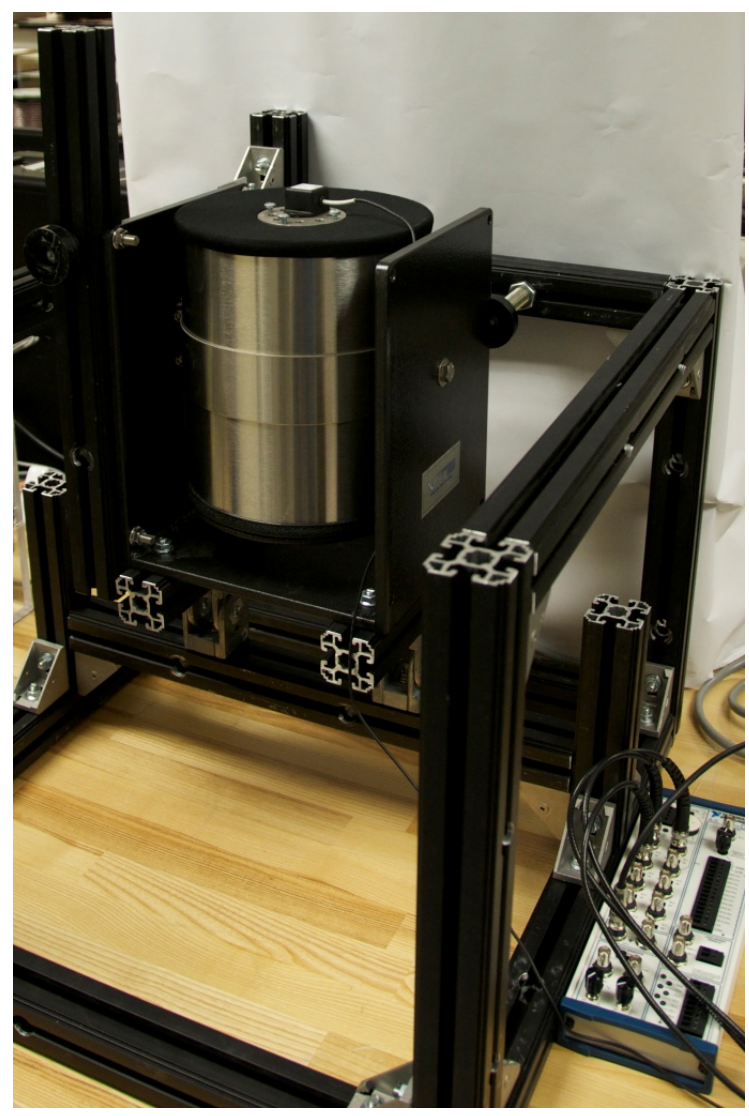

Fig. 9. Vibration Test Stand

Based upon research and past experiments, a set of frequencies and corresponding accelerations were chosen. The spectrum applied to the batteries can be seen in Table II. The spectrum chosen covers a wide range of frequencies at various accelerations that would provide ample energy to excite any possible natural frequencies and simulate vibrational levels comparable to a launch event. The frequencies and amplitudes of these vibrations are simultaneously produced and recorded using a LabView user interface with onboard accelerometers to monitor vibrations endured by the battery pack. When tested, batteries are rigidly attached to the vibratory test bed, and the spectrum applied to each axis of the batteries (x,y and z) for 60 seconds. Fig. 10 shows the gui which is used to alter test parameters and examine the resultant outputs.

After this test the batteries are then checked to ensure full charge capabilities and for external damage and possible internal short-circuiting damage. This is 


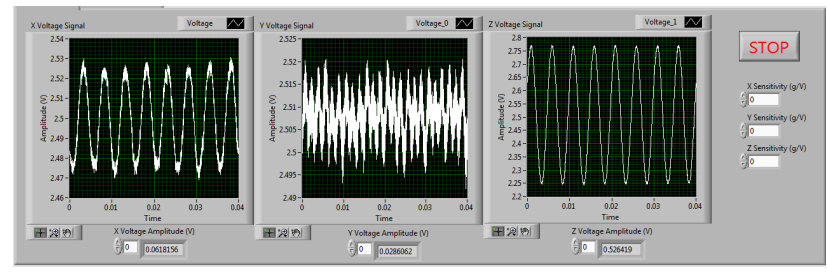

Fig. 10. Vibration Certification Test Run

TABLE II

VIBRATION SPECTRUM DETAILS

\begin{tabular}{llll}
\hline Frequency & $\mathrm{GSD}\left(\left(G^{2}\right) / \mathrm{Hz}\right.$ & $\mathrm{db} / \mathrm{OCT}$ & $\mathrm{G}_{r m s}$ \\
\hline 20.00 & 0.028800 & 0.0 & 0.0 \\
40.00 & 0.028800 & 0.00 & 0.76 \\
70.00 & 0.072000 & 4.93 & 1.43 \\
700.00 & 0.072000 & 0.00 & 6.89 \\
2000.00 & 0.018720 & -3.86 & 9.65 \\
\hline
\end{tabular}

further validated by running the charge/discharge profile discussed in section IV-A. If any of the previously mentioned capabilities are deteriorated significantly by vibrational exposure, or if external damage has altered the form of the batteries beyond specifications, or if external damage inhibits the use of the batteries, then the batteries are considered to have failed and need to be replaced.

\section{CONClusion}

In this paper, we presented a combination of different testbeds and experiments which are used to certify offthe-shelf 18650 lithium ion batteries for small satellite missions. Each of the tests conducted simulated operating conditions and scenarios which cells and battery packs may be subjected to once launched. It is very necessary from the safety perspective to verify the operations and functionality of these components to avoid any malfunctions which might have a direct impact on the mission. The developed testbeds satisfy NASA certification standards for the current battery technologies used for small satellite missions.

In the future we plan to further generalize the testbeds for certification of other types of cells. Especially the vibration test stand needs to be able to accommodate heavy battery packs with varying dimensions and the short circuit testbed needs to have higher current carrying capability within safe operating margins. The goal of this work is to set up a standardized procedure for these certification methods which could be easily reproduced in a relatively short span of time despite minor changes in cell geometry and electrochemistry as well as mission requirements.

\section{REFERENCES}

[1] M. D. D. Staff, "Small spacecraft technology state of the art," Ames Research Center,MoffettField, California, Tech. Rep., 2014.

[2] J. Jeevarajan, "Hazards, safety and design considerations for commercial lithium-ion cells and batteries," in 2nd International Association for Advancement of Space Safety Conference, May 2007.

[3] —, "Safety limitations associated with commercial 18650 lithium-ion cells," in Lithium Mobile Power and Battery Safety, 2010.

[4] M. Daigle and C. Kulkarni, "Electrochemistry-based battery modeling for prognostics," in Annual Conference of the Prognostics and Health Management Society 2013, Oct. 2013, pp. 249-261.

[5] D. Doughty and E. P. Roth, "A general discussion of li ion battery safety," Electrochemical Society Interface, vol. 21, no. 2, pp. 3744, 2012.

[6] B. McKissock, P. Loyselle, and E. Vogel, Guidelines on Lithiumion Battery Use in Space Applications, Glenn Research Center, Cleveland, Ohio, 2009.

[7] D. Belov and M.-H. Yang, "Failure mechanism of li-ion battery at overcharge conditions," Journal of Solid State Electrochemistry, vol. 12, no. 7-8, pp. 885-894, 2008.

[8] J. A. Jeevarajan and C. S. Winchester, "Battery safety qualifications for human ratings," Electrochemical Society Interface, vol. 21 , no. 2, pp. 51-55, 2012.

[9] J. Jeevarajan, "Hazards associated with high voltage high capacity lithium-ion batteries," Electrochemical Society Interface, vol. 33, no. 22, pp. 1-6, 2011. 